\title{
Genomic identification and characterization of MYC family genes in wheat (Triticum aestivum L.)
}

\author{
Jian-fang Bai ${ }^{1,2 \dagger}\left(\mathbb{D}\right.$, Yu-kun Wang ${ }^{3+} \mathbb{D}$, Li-ping Guo ${ }^{1,2,4+}$, Xiao-ming Guo ${ }^{1,2}$, Hao-yu Guo ${ }^{1,2}$, Shao-hua Yuan ${ }^{1,2}$, \\ Wen-jing Duan ${ }^{1,2}$, Zihan Liu ${ }^{1,2}$, Chang-ping Zhao ${ }^{1,2^{*}}$, Feng-ting Zhang ${ }^{1,2}$ and Li-ping Zhang ${ }^{1,2^{*}}$ (D)
}

\begin{abstract}
Background: MYC transcriptional factors are members of the $6 H L H$ (basic helix-loop-helix) superfamily, and play important roles in plant growth and development. Recent studies have revealed that some MYCs are involved in the crosstalk between Jasmonic acid regulatory pathway and light signaling in Arabidopsis, but such kinds of studies are rare in wheat, especially in photo-thermo-sensitive genic male sterile (PTGMS) wheat line.

Results: 27 non-redundant MYC gene copies, which belonged to 11 TaMYC genes, were identified in the whole genome of wheat (Chinese Spring). These gene copies were distributed on 13 different chromosomes, respectively. Based on the results of phylogenetic analysis, 27 TaMYC gene copies were clustered into group I, group III, and group IV. The identified TaMYC genes copies contained different numbers of light, stress, and hormone-responsive regulatory elements in their 1500 base pair promoter regions. Besides, we found that TaMYC3 was expressed highly in stem, TaMYC5 and TaMYC9 were expressed specially in glume, and the rest of TaMYC genes were expressed in all tissues (root, stem, leaf, pistil, stamen, and glume) of the PTGMS line BS366. Moreover, we found that TaMYC3, TaMYC7, TaMYC9, and TaMYC10 were highly sensitive to methyl jasmonate (MeJA), and other TaMYC genes responded at different levels. Furthermore, we confirmed the expression profiles of TaMYC family members under different light quality and plant hormone stimuli, and abiotic stresses. Finally, we predicted the wheat microRNAs that could interact with TaMYC family members, and built up a network to show their integrative relationships.

Conclusions: This study analyzed the size and composition of the MYC gene family in wheat, and investigated stress-responsive and light quality induced expression profiles of each TaMYC gene in the PTGMS wheat line BS366. In conclusion, we obtained lots of important information of TaMYC family, and the results of this study was supposed to contribute novel insights and gene and microRNA resources for wheat breeding, especially for the improvement of PTGMS wheat lines.
\end{abstract}

Keywords: Wheat, MYC, Gene family, JA signaling, Light response, Male sterile

\section{Background}

The Jasmonic acid (JA) signaling pathway is complicated and is involved in several regulatory processes, such as plant growth and development, fertility regulation, and plant immunity [1, 2]. In Arabidopsis, components of JA signaling pathway include F-box protein CORONATINE

\footnotetext{
*Correspondence: cp_zhao@vip.sohu.com; Ipzhang8@126.com

†Jian-fang Bai, Yu-kun Wang and Li-ping Guo contributed equally to this work.

'Beijing Engineering Research Center for Hybrid Wheat, Beijing Academy of Agriculture and Forestry Sciences, Beijing 100097, China

Full list of author information is available at the end of the article
}

INSENSITIVE1 (COI1), Jasmonate-ZIM (JAZ) domain repressor, and the bHLH transcription factor MYC2, which can regulate the expression patterns of JA-response genes [3]. JAZ proteins have been shown to block MYC2 activity in the absence of bioactive JAs by recruiting the general corepressors TOPLESS (TPL) and TPL-related proteins through the interaction with the adaptor protein novel interactor of JAZ (NINJA) [4]. In Arabidopsis, MYC2 was the first transcription factor (TF) found to be regulated by the JAZ proteins [3], and involved in defense regulation against insect herbivory via a partially redundant manner

(c) The Author(s). 2019 Open Access This article is distributed under the terms of the Creative Commons Attribution 4.0 International License (http://creativecommons.org/licenses/by/4.0/), which permits unrestricted use, distribution, and reproduction in any medium, provided you give appropriate credit to the original author(s) and the source, provide a link to the Creative Commons license, and indicate if changes were made. The Creative Commons Public Domain Dedication waiver (http://creativecommons.org/publicdomain/zero/1.0/) applies to the data made available in this article, unless otherwise stated. 
with its homologs MYC3 and MYC4 $[5,6]$. These three MYC TFs can form homo- and heterodimers to bind Gbox (CACGTG) elements or some variants of G-box elements [6]. However, these three MYC TFs play different roles in signaling pathways, despite they share the similar DNA binding sites. For instance, MYC3 and MYC4 are important for JA-mediated resistance to the herbivore Spodoptora littoralis, while the roles of MYC2, MYC3 and MYC4 are very weak in regulating JA-mediated inhibition of primary root growth. These differences might due to their preferential production in root and shoot tissues [5, 6]. In addition to the JA signaling pathway, MYC2 is also involved in repressing primary root growth, anthocyanin biosynthesis, oxidative stress tolerance [7], blue lightmediated photomorphogenic growth, resistance to necrotrophic fungi, and biosynthesis of tryptophan and indoleglucosinolates [8]. Therefore, MYC2 acts as a key factor that connects many pathways.

Among environmental signals, light is an important influential factor modulating plant growth and development. Light is a source of energy for plant photosynthesis and acts as a signal in the coordination of plant adaptive responses to environmental changes [9]. Plant responses to light are often mediated by photoreceptors, which are sensitive to specific wavelengths of the solar spectrum. Phytochromes belong an important class of photoreceptors play roles in light signaling. Phytochrome B (PHYB) is mainly sensitive to red light ( $R$; wavelength: $660 \mathrm{~nm}$ ) and PHYA is primarily sensitive to far-red light (FR; wavelength: $730 \mathrm{~nm}$ ) radiation [10]. In Arabidopsis, under farred light or darkness, plants show etiolation and elongated hypocotyls phenotypes, but the hypocotyl elongation is inhibited under red light conditions, which indicates that PHYA acts as a negative regulator of skotomorphogenesis, and PHYB is a positive regulator of photomorphogenesis [11]. Phytochromes proteins mainly include two types: the red light absorbing type (known as Pr) and the far-red light absorbing type (known as Pfr). They are interchangeable based on the R:FR ratios in environments [12]. Low $\mathrm{R}: F R$ ratio can reduce the levels of Pfr, and may be involved in shade-avoidance syndrome [11]. Recently, some studies have shown that components of JA signaling pathway, such as JAZ proteins, COIs and MYC2, are involved in several light-mediated responses. Robson et al. (2010) found that Arabidopsis mutations jin 1 and $m y c 2$ are more sensitive to shade or FR light, and displayed an elongated hypocotyl phenotype under low R:FR ratio than wild type [13]. In addition, light-response related genes were upregulated under FR and blue light (BL) conditions in jin1/ myc2 mutant [14]. MYC2 could interact with the Z-box and G-box light-response elements and was thought as a negative regulator of blue light-mediated photomorphogenic growth [14]. Furthermore, MYC2 and SPA1 (suppressor of PHYA) may act redundantly in the dark and synergistically under light to suppress photomorphogenesis [15].

In addition, MYC2 also participates in the crosstalk among JAs and other plant hormones. In Arabidopsis, MYC2 was characterized as a transcriptional activator in ABA-inducible gene expression [16]. Song et al. (2014) demonstrated that MYC2 can interact with ethylenestabilized transcription factor EIN3 and modulated JA and ET signal antagonism in Arabidopsis [17].

Wheat is one of the most important food crops. The fertility of the PTGMS wheat line BS366 is controlled by temperature and/or photoperiods [2]. The pollen of BS366 can not be fully spilled out, due to the impaired anther dehiscence, which can be recovered by the application of MeJA in vitro [2]. In wheat JA signaling pathway, $8 \mathrm{COI}$ genes and $14 J A Z$ genes have been identified $[2,18]$. However, few studies have been reported about wheat $M Y C$ gene family, which is an important component of the JA signaling pathway. In the present study, the TaMYC gene family was characterized using the latest genome sequences. We analyzed gene structures, conserved motifs, chromosome localization, and the regulatory networks of the TaMYC gene family. Given the important roles of microRNAs, such as miR1120 and miR1130 are involved in the JA signaling pathway and participate in anther development in wheat PTGMS line [19], we also predicted the interactive relationships between TaMYC and microRNAs. In addition, the expression profiles of TaMYC genes in the PTGMS wheat line BS366 were detected using qRT-PCR. Results in this study are expected to support a basis for further investigations on the functions of TaMYC genes, and provide some gene resources for revealing the molecular mechanisms of male sterility in PTGMS wheat.

\section{Results \\ Identification of TaMYC gene family}

After the removal of redundant gene, 27 non-redundant $M Y C$ gene copies, which belonged to 11 MYC genes, were identified. Firstly, we monitored the physical and chemical characteristics of these $M Y C$ gene copies. The coding sequence lengths of $27 M Y C$ gene copies were ranged from $1332 \mathrm{bp}$ to $2088 \mathrm{bp}$, and the deduced protein lengths were ranged from 443 to 695 amino acids (Table 1 ). The predicted molecular weights (MWs) of each MYC protein were ranged from $47.53 \mathrm{kDa}$ to $75.62 \mathrm{kDa}$, and the corresponding isoelectric points (IPs) were changed from 4.96 to 8.73 (Table 1). Subcellular localization predictions revealed that MYC proteins were functioned in chloroplast, cytoplasmic, nuclear, or plasma membrane (Table 1). Different characteristics of TaMYC genes and proteins were obtained, and the results indicated that different TaMYC proteins might have different biological functions. 
Table 1 Characteristics of TaMYC gene family members

\begin{tabular}{|c|c|c|c|c|c|c|}
\hline Gene name & Sequence ID & Locations & Protein/AA & Isoelectric point & $\begin{array}{l}\text { Molecular weight } \\
\text { of decuced protein/KD }\end{array}$ & Subcellular Localization \\
\hline TaMYC1-A & TraesCS1A02G102400 & 1A:98584808:98591571:+ & 626 & 5.65 & 68.48 & Nuclear \\
\hline TaMYC2-A & TraesCS1A02G193200 & 1A:349358465:349360851:+ & 693 & 6.37 & 74.04 & Nuclear \\
\hline TaMYC1-B1 & TraesCS1B02G112900 & 1B:131456949:131464291:+ & 631 & 5.84 & 69.22 & Nuclear \\
\hline TaMYC1-B2 & TraesCS1B02G113100 & 1B:131775632:131785030:+ & 688 & 5.87 & 75.62 & Nuclear \\
\hline TaMYC2-B & TraesCS1B02G208000 & 1B:376071131:376073212:+ & 693 & 6.15 & 75.09 & Nuclear \\
\hline TaMYC2-D & TraesCS1D02G196900 & 1D:277092891:277094978:+ & 695 & 6.15 & 75.35 & Nuclear \\
\hline TaMYC3-A1 & TraesCS2A02G409400 & 2A:667011017:667015600:+ & 568 & 5.37 & 62.65 & Chloroplast|Cytoplasmic \\
\hline TaMYC3-A2 & TraesCS2A02G409600 & 2A:667647129:667652609:+ & 558 & 5.54 & 60.82 & Chloroplast|Cytoplasmic \\
\hline TaMYC3-B & TraesCS2B02G428000 & 2B:615352721:615356928:+ & 573 & 5.17 & 62.77 & Chloroplast \\
\hline TaMYC7-D & TraesCS2D02G406800 & 2D:522323574:522329854:+ & 465 & 4.96 & 51.53 & Cytoplasmic \\
\hline TaMYC3-D & TraesCS2D02G406900 & 2D:522521895:522526799:+ & 567 & 5.19 & 61.82 & Cytoplasmic|Chloroplast \\
\hline TaMYC8-D & TraesCS2D02G575600 & 2D:639529624:639535060:- & 589 & 5.24 & 66.17 & Cytoplasmic \\
\hline TaMYC4-A & TraesCS3A02G158600 & 3A:156267999:156270204:- & 616 & 6.52 & 67.35 & Nuclear|Cytoplasmic \\
\hline TaMYC5-A & TraesCS3A02G252900 & 3A:474209127:474210506:- & 459 & 8.43 & 49.17 & Cytoplasmic|Mitochondrial \\
\hline Gene name & Sequence ID & Locations & Protein/AA & Isoelectric point & $\begin{array}{l}\text { Molecular weight } \\
\text { of decuced protein/KD }\end{array}$ & Subcellular Localization \\
\hline TaMYC4-B & TraesCS3B02G185400 & 3B:201339407:201341777:- & 625 & 7.11 & 68.24 & Nuclear|Cytoplasmic \\
\hline TaMYC5-B1 & TraesCS3B02G288700 & 3B:463203072:463205129:+ & 443 & 8.47 & 47.53 & Cytoplasmic|Mitochondrial \\
\hline TaMYC5-B2 & TraesCS3B02G284800 & 3B:456323130:456324920:- & 475 & 7.77 & 50.76 & Cytoplasmic|Mitochondrial \\
\hline TaMYC4-D & TraesCS3D02G166300 & 3D:138192597:138194964:- & 625 & 6.76 & 68.31 & Nuclear|Cytoplasmic \\
\hline TaMYC5-D & TraesCS3D02G253700 & 3D:355318384:355320162:- & 465 & 8.73 & 49.82 & Mitochondrial|Cytoplasmic \\
\hline TaMYC6-A & TraesCS4A02G028900 & 4A:21063512:21065305:- & 597 & 6.56 & 65.55 & Nuclear|Cytoplasmic \\
\hline TaMYC6-B & TraesCS4B02G276900 & 4B:558578472:558580268:+ & 598 & 6.73 & 65.56 & Nuclear|Cytoplasmic \\
\hline TaMYC8-B & TraesCS4B02G397400 & 4B:671703616:671707933:+ & 564 & 5.18 & 62.86 & Chloroplast|Cytoplasmic \\
\hline TaMYC9-B & TraesCS4B02G397800 & 4B:671764988:671776128:- & 510 & 5.47 & 57.00 & Plasma Membrane|Nuclear \\
\hline TaMYC10-D & TraesCS4D02G224600 & 4D:381551243:381559778:+ & 557 & 6.49 & 61.40 & Mitochondrial|Cytoplasmic \\
\hline TaMYC6-D & TraesCS4D02G275500 & 4D:446428380:446430176:+ & 598 & 6.56 & 65.59 & Nuclear|Cytoplasmic \\
\hline TaMYC11-A1 & TraesCS5A02G489500 & 5A:659338425:659342323:- & 570 & 5.31 & 63.15 & Cytoplasmic|Mitochondrial \\
\hline TaMYC11-A2 & TraesCS5A02G558500 & 5A:709193171:709197273:+ & 585 & 5.26 & 64.93 & Cytoplasmic \\
\hline
\end{tabular}

Analysis of chromosomal locations and synteny

In order to understand the relative position of each TaMYC gene copy on wheat chromosomes, we marked their physical placements on wheat A, B, and D chromosomes. As shown in Figs. 1, 27 TaMYC gene copies were located on 13 chromosomes. 9, 10, and 8 TaMYC gene copies were located on chromosomes 1A-5A, 1B-4B, and 1D-4D, respectively (Fig. 1).

Gene and chromosomal segment duplication are the major forces of genome evolution in plants [20]. In the wheat genome, four tandem duplication events (TaMYC1B1/TaMYC1-B2, TaMYC3-A1/TaMYC3-A2, TaMYC5-B1/ TaMYC5-B2, and TaMYC11A-1/TaMYC11A-2) and 25 segmental duplication events were identified, which indicate that segmental duplication events were the main cause of the increase of $M Y C$ members in wheat (Additional file
1: Figure S1and Additional file 7: Table S4). Synteny analysis among TaMYCs and its ancestors were also analyzed. Nine members (TaMYC1-A, TaMYC3-A2, TaMYC4-A, Ta MYC6-A, TaMYC2-D, TaMYC3-D, TaMYC4-D, TaMYC5$D$ and TaMYC6-D) of the TaMYC gene family have homology with genes of T. urartu and Ae. tauschii (Additional file 1: Fig. S1).

\section{Phylogenetic analysis of TaMYC proteins}

To reveal the functional information of TaMYC genes, a phylogenetic tree, which based on the compare among wheat, Arabidopsis, and rice, was constructed using N-J method. As shown in Fig. 2, MYC proteins of three species were clustered into four groups (I, II, III, and IV). TaMYCs were distributed in groups I, III and IV. Seven TaMYC proteins (16 copies) were clustered into group I, 


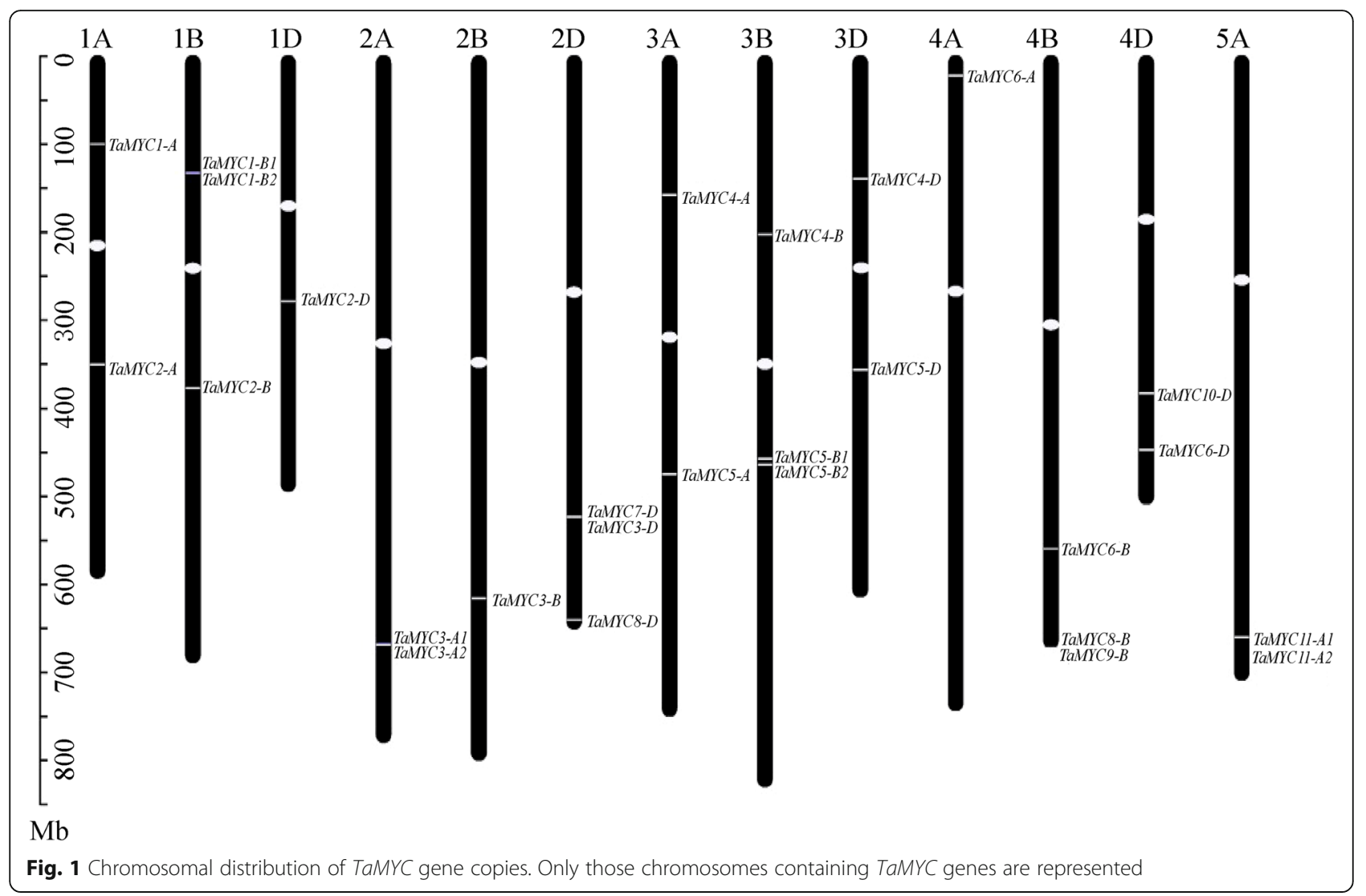

while only TaMYC2 (three copies) was clustered into Group III. It was worth noting that three copies of TaMYC2 had a close homology with AtMYC2, AtMYC3 and AtMYC4, which are genes that have been demonstrated to play similar roles in plant development (Fig. 2).

\section{Structural analysis of TaMYC genes and proteins}

For understanding the structural features of TaMYC family members, firstly, the exon-intron structural features were revealed by aligning the predicted CDS against corresponding genomic sequences. As shown in Fig. 3, the intron-exon structures of different TaMYC genes were diverse, while copies of the same genes were similar or same, such as TaMYC6 (TaMYC6-A, TaMYC6$B$ and TaMYC6-D) and TaMYC11 (TaMYC11-A1 and TaMYC11-A2). It was notable that $M Y C$ genes of the same subgroup shared similar intron-exon structures, for instance, TaMYC4 and TaMYC6 were both in subgroup IV, and they only had one exon (Fig.2 and Fig. 3).

Motifs in TaMYC proteins were also predicted in this study. Similar with the intron-exon structures of TaMYC genes, proteins of the same subgroup shared the same or similar motifs. Most copies of TaMYC proteins possessed six, seven or eight motifs (Fig. 3). TaMYC7-D only had five motifs (Fig. 3). As the member of bHLH superfamily, MYC family proteins contain a
bHLH domain and a conserved bHLH-MYC N domain $[15,21]$. In this study, motifs $2,3,6$ and 7 corresponded to bHLH-MYC_N domains, while motifs 1 and 4 corresponded to bHLH domains (Additional file 2: Fig. S2). The biological functions of other motifs remains unknown, but it could be predicted that some TaMYC proteins had unknown functions.

\section{Analysis of cis-regulatory elements of TaMYC genes}

The upstream promoter regions (1500 bp, Additional file 3: File S1) of TaMYC gene copies were retrieved from the wheat genome to identify cis-regulatory elements. Five light-responsiveness regulatory elements, including ACE, ATC-motif, Box 4, G-box and MRE were identified in all TaMYC promoter regions, indicating that TaMYCs might be involved in light signaling pathways (Fig. 4 and Additional file 5: Table S2). Eight hormone-responsive regulatory elements, including TGA-element, TCAelement, TATC-box, P-box, GARE-motif, CGTCA/ TGACG-motif, AuxRR-core, and ABRE, which were associated with auxin, salicylic acid, gibberellin, MeJA, and $\mathrm{ABA}$ responses, were identified in the promoter region of TaMYC copies (Fig. 4 and Additional file 5: Table S2). Besides, six stress-responsive regulatory elements, WUNmotif, TC-rich repeats, MBS, LTR, GC-motif and ARE, which were associated with wound responsiveness, 


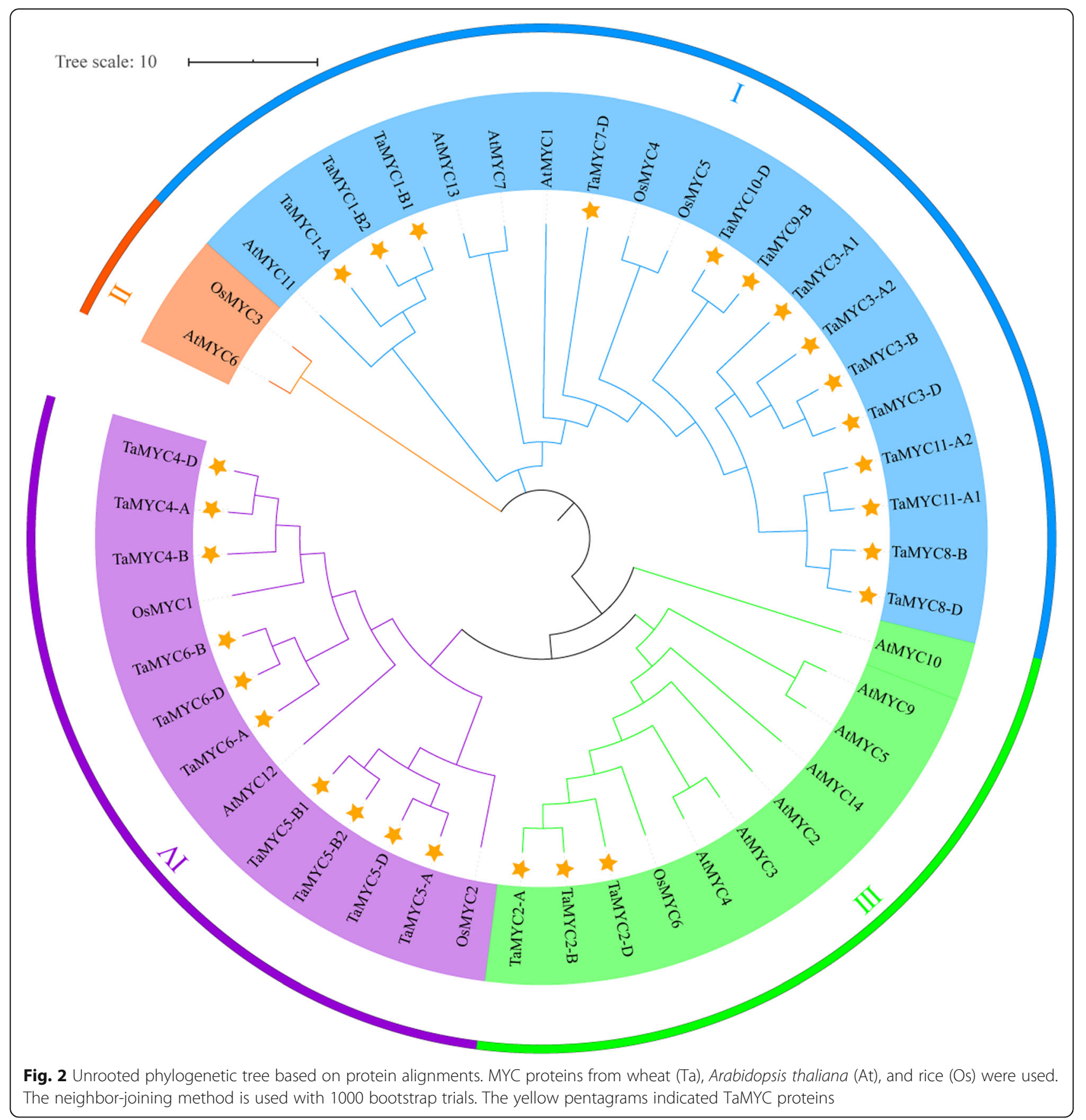

defense and stress responsiveness, drought-inducibility, low-temperature responsiveness, anoxic specific inducibility and anaerobic induction, respectively, were identified (Fig. 4 and Additional file 5: Table S2). In addition, cisregulatory elements for five regulators of development regulation and two regulators of biosynthesis regulation were identified. As shown in Additional file 5: Table S2, different types and numbers of regulatory elements were identified in the promoter regions of different TaMYC genes, indicating that TaMYC genes might have different functions in stress resistance, growth and development.
Tissue/organ-specific expression profiles of TaMYC genes To study the tissue/organ-specific expression patterns of the 11 identified TaMYC genes, their expression patterns in root, stem, leaf, petal, pistil, stamen, and glume of the PTGMS wheat line BS366 were investigated by qRT-PCR. As shown in Figs. 5, 11 TaMYC genes showed different expression levels in different tissues. The expression levels of TaMYC5 and TaMYC9 in glume were higher than that in other tissues (Fig. 5). In addition, TaMYC1 and TaMYC2 had relatively high expression levels in pistil tissue, while TaMYC6, TaMYC10, and TaMYC11 had 


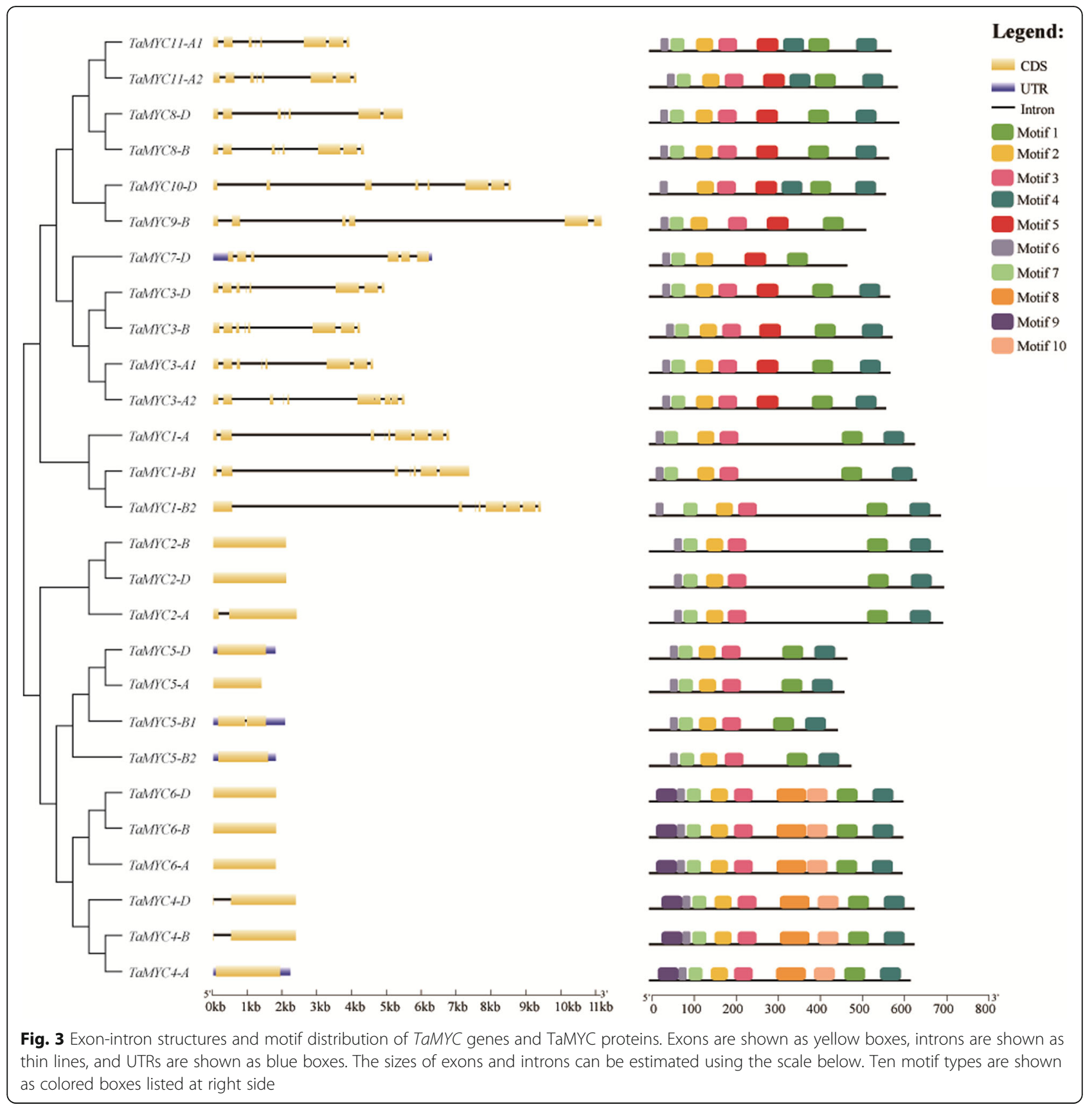

relatively high expression in leaf tissue (Fig. 5). TaMYC4 was constitutively expressed in all six tissues (Fig. 5). Meanwhile, we noticed that TaMYC2, TaMYC4, TaM YC10, and TaMYC11 displayed relatively high expression levels in stamen (Fig. 5).

\section{Effects of exogenetic MeJA treatment}

In order to investigate the functions of MYCs in the JA signaling pathway at anther dehiscent stage, the expression profiles of TaMYC genes in anthers, which treated with different concentrations of MeJA, were analyzed. As shown in Fig. 6, the expression of TaMYC3 was high under $2 \mathrm{mmol} / \mathrm{L}$ MeJA. The expression levels of TaMYC5, TaMYC7, TaMYC8, TaMYC9, and TaMYC10 in anther were induced by $4 \mathrm{mmol} / \mathrm{L}$ MeJA (Fig. 6). These results indicate that these TaMYC genes could be strongly induced by MeJA and might be function on the JA signaling pathway in anther of the PTGMS wheat line BS366.

\section{Photochromic conversion-induced expression profiles of TaMYC genes}

Many studies have suggested that MYC may be involved in the cross-talk between the JA signaling pathway and 


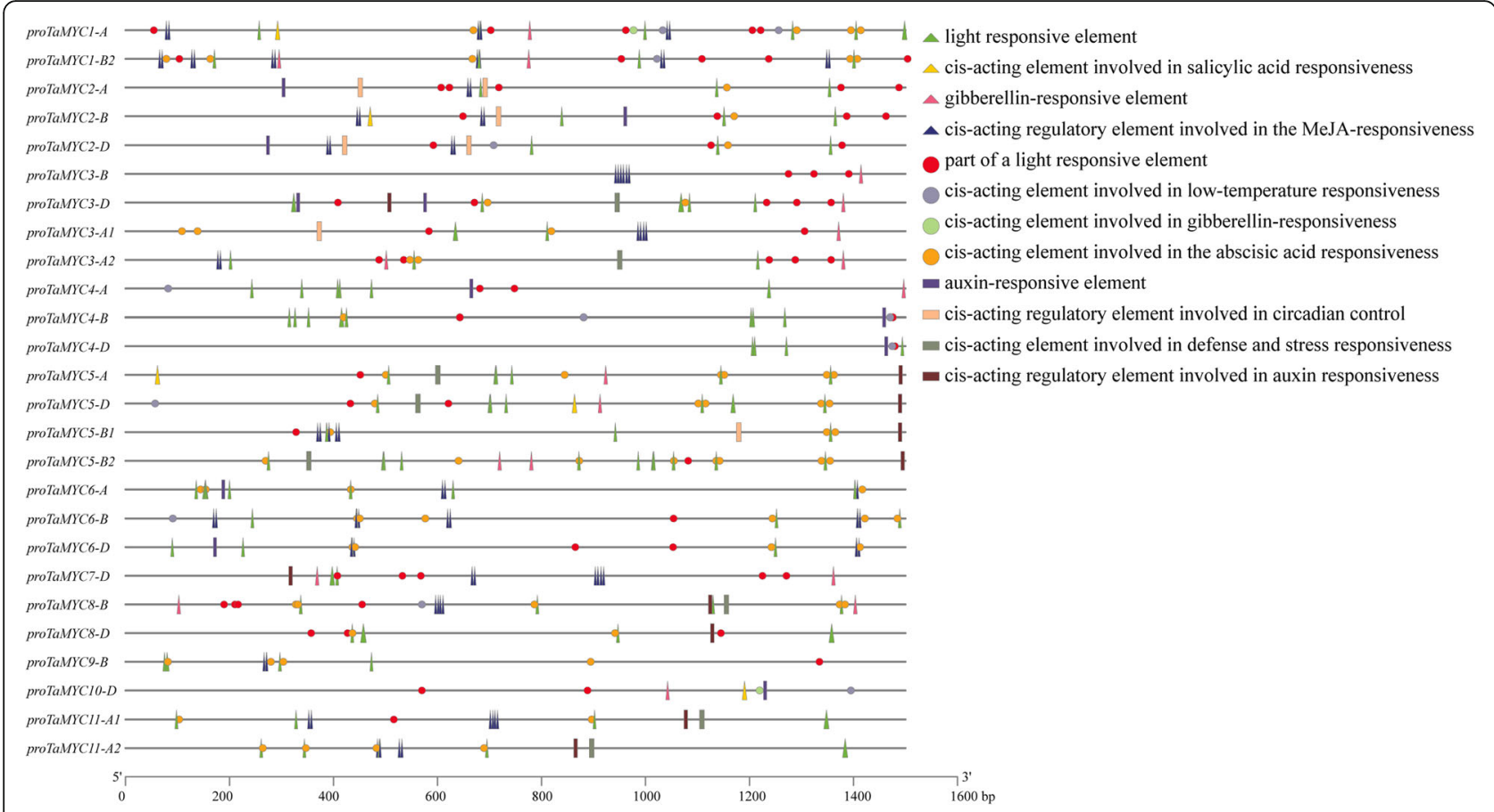

Fig. 4 The number and composition of cis-acting regulatory elements in the promotor region of TaMYC genes. 1500 base pair promoter region of each gene copy is displayed. Different colorful shapes show different elements

light signaling pathway (R:FR ratio mediated Pfr/Pr conversion) [12]. To investigate photochromic conversioninduced expression profiles of TaMYC genes, different R: FR ration light treatments were performed on BS366 seedlings. As shown in Fig. 7, TaMYC1, TaMYC2, TaMYC6, TaMYC7, TaMYC8, TaMYC9 and TaMYC11 were upregulated in R and FR light-enriched conditions compared to white light conditions. In addition, TaMYC4 was downregulated by far-red light, and TaMYC5 and TaMYC10 were inhibited under $\mathrm{R}$ light condition (Fig. 7).

\section{Expression profiles of TaMYC genes under phytohormone treatments}

The expression profiles of TaMYC genes in the leaf tissues of the PTGMS line BS366 under plant hormones treatments were analyzed to determine the responsive profiles. Under ABA treatment, the expression levels of TaMYC2, TaMYC8, and TaMYC9 were upregulated at $4 \mathrm{~h}$ posttreatment, then downregulated at $12 \mathrm{~h}$ post-treatment (Fig. 8). TaMYC4, TaMYC5 and TaMYC6 were induced after ABA treatment. TaMYC1, TaMYC3, TaMYC7, TaMYC10 and TaMYC11 showed negative responses to ABA treatment (Fig. 8). Under GA treatment, the expression of TaMYC7, TaMYC8, TaMYC9, and TaMYC10 were inhibited, while the expression of TaMYC2, TaMYC4, TaMYC5, and TaMYC6 were promoted (Fig. 8). The transcript profiles of TaMYC3 and TaMYC11 were slightly upregulated and peaked at $4 \mathrm{~h}$ post-treatment. TaMYC1 was downregulated at $4 \mathrm{~h}$ post-treatment, but showed an increase profile at $12 \mathrm{~h}$ post-treatment (Fig. 8). Under IAA treatment, the expressions of all TaMYC genes were downregulated (Fig. 8).

\section{Abiotic stress-induced expression profiles of TaMYC genes}

The transcriptional profiles of TaMYC genes under abiotic stresses were monitored in this study. As shown in Fig. 9, the expressions of TaMYC1, TaMYC2, TaMYC3, TaMYC4, TaMYC5, TaMYC6, TaMYC8, TaMYC10, and TaMYC11 were upregulated under low temperature treatment. The expression levels of TaMYC7 and TaMYC9 were decreased at $4 \mathrm{~h}$ post-treatment, and recovered at $12 \mathrm{~h}$ post-treatment (Fig. 9). Under high salinity conditions, TaMYC1, TaMYC2, TaMYC3, ТaMYC6, TaMYC7, ТaM YC8, TaMYC9, TaMYC10, and TaMYC11 were downregulated. Only TaMYC4 and TaMYC5 were promoted after saline treatment (Fig. 9). Under drought stress, TaMYC5 and TaMYC8 were upregulated, while the rest of TaMYCs were downregulated (Fig. 9).

\section{microRNA targeting prediction of TaMYC genes}

In order to uncover the interactions between microRNAs (miRNAs) and their MYC targets, we predicted the potential networks using online tool (Additional file 6: Table S3). As shown in Fig. 10, seven TaMYC genes, including TaMYC3, TaMYC5, TaMYC6, TaMYC7, TaMYC8, TaMYC10, TaM YC11, were regulated by 12 miRNAs (taemiR1127b-3p, taemiR9657a-3p, taemiR9676-5p, taemiR1138, taemiR167b, taemiR5384-3p, taemiR9773, taemiR1128, taemiR164, 

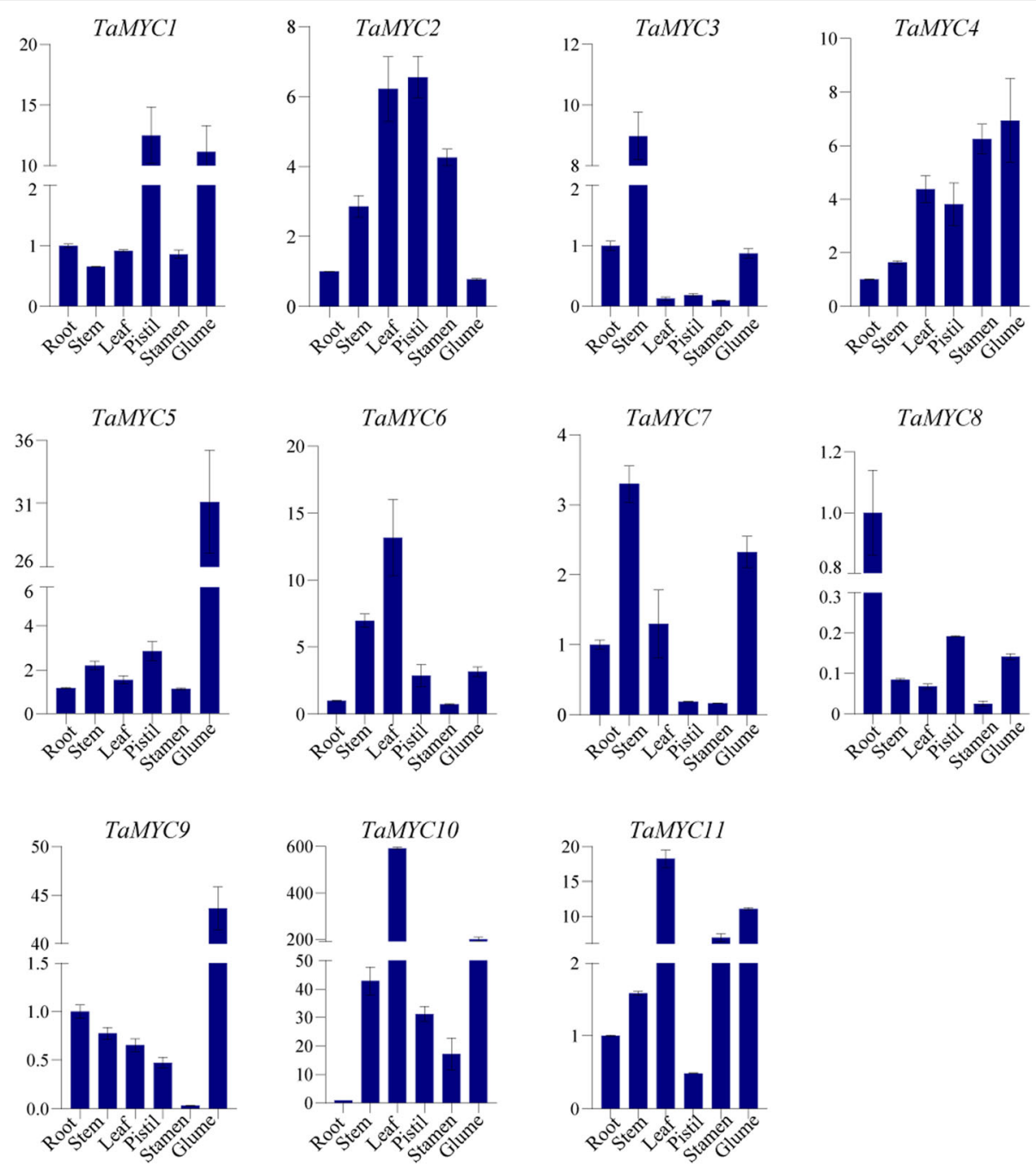

Fig. 5 Relative expression of TaMYC genes in six wheat tissues (root, stem, leaf, pistil, stamen, and glume) in the heading stage by qRT-PCR. The error bars indicate the standard deviation obtained from three replications. Wheat actin gene is used as the inner reference

taemiR9657c-3p, taemiR9675-3p, and taemiR9677a). We speculated these miRNAs could inhibit the expression levels of target TaMYCs at the transcriptional or translational level. One TaMYC gene might be targeted by multiple miRNAs, while several TaMYC genes might be regulated by the same miRNA (Fig. 10). In order to understand the potential functions, we pointed out the response model of each target TaMYC gene under different stresses or treatments. TaMYC3, TaMYC5, TaMYC6, and TaMYC8 were responded to cold stress, TaMYC7 was mainly responded to salinity, and TaMYC10 and TaMYC11 were thought to show a close relationship with drought stress (Fig. 10). Only TaMYC3 showed a clear response to $2 \mathrm{mmol} / \mathrm{L}$ MeJA treatment, and the rest of 6 TaMYCs were promoted at $4 \mathrm{mmol} /$ L MeJA treatment (Fig. 10). TaMYC7-D was sensitive to red light stimulus, while others were more sensitive to far red light (Fig. 10). Besides, we found that TaMYC3 and TaMYC8 were regulated by ABA, TaMYC5 and TaMYC6 could response to GA, and TaMYC7, TaMYC10, and TaMYC11 were mainly regulated by IAA (Fig. 10). Although the precise functions of most miRNAs in this study were unknown, some miRNAs (such as taemiR167 and taemiR1127b-3p) had already identified as factors involved in the regulation of male sterility in wheat $[2,19]$. Based on the network shown in Fig. 10, we thought that TaMYC3, TaMYC5, TaMYC6, and TaMYC8 maintained high possibilities on the regulation of temperature-induced male sterility in wheat PTGMS line BS366.

\section{Discussion}

The JA signaling pathway is involved in plant growth and development, and it can regulate the plant fertility [22], anthocyanin accumulation [23] and leaf senescence [24]. MYC is an important component of the COI1/ JAZ/MYC2 complex and can mediate JA and various hormone signaling pathways, and the light signaling 

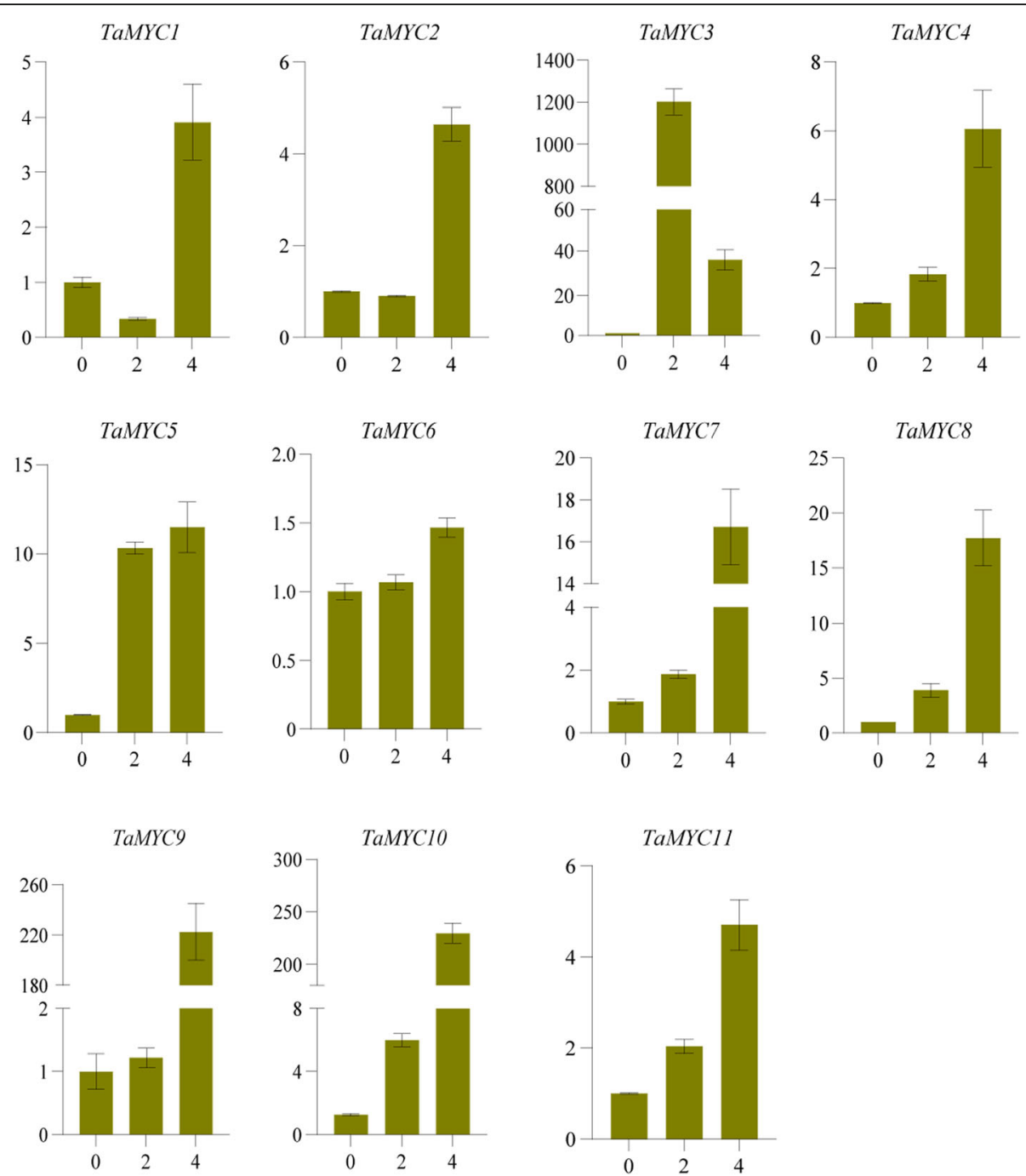

Fig. 6 Relative expression of TaMYC genes in anthers treated with different concentrations of MeJA. Values on the x-axis indicate the MeJA concentration. MeJA: spikelets were treated with MeJA (0 mM, 2 mM, and $4 \mathrm{mM})$. The error bars indicate the standard deviation obtained from three replications. Wheat Actin gene is used as the inner reference

pathway [12]. However, there are few reports regarding the function of MYC in crop plants. In the present study, aiming to understand the roles of $M Y C$ genes in wheat, 27 candidate TaMYC copies were isolated (Fig. 1 and Table 1) and clustered into $11 M Y C$ genes (Fig. 2). These TaMYC genes were classified into 3 subgroups based on phylogenetic analysis and shared relatively close homology with MYCs of Arabidopsi and rice (Fig. 2). Furthermore, phylogenetic analysis revealed that TaMYC2 had close homology with AtMYC2, AtMYC3 and AtMYC4, indicating that TaMYC2 may have similar roles in regulating wheat development (Fig. 2).

Cis-regulatory sequences, such as enhancers and promoters, can control development and physiology by regulating gene expression [25]. Cis-regulatory elements, acting as important molecular switches, are involved in the regulation of gene transcription under external stimuli [26]. To clarify the functions of TaMYC genes, cis-regulatory elements in the promoter regions of TaMYC genes, and their expression profiles under various stresses were analyzed. It was found that six stress-responsive elements, five lightresponsive elements, and eight hormone-responsive elements were identified in TaMYC promoter regions (Fig. 4 and Additional file 6: Table S3). The 11 TaMYCs contained different types and numbers of cis-acting regulatory elements in each promoter region, indicating that these genes had different regulatory functions that responded to different stress and hormone treatments. Furthermore, we evaluated the expression profiles of TaMYC genes in PTGMS wheat line BS366 seedlings under different stress conditions and hormone treatments. Inducible expression analyses revealed that both TaMYC4 and TaMYC6, with similar 

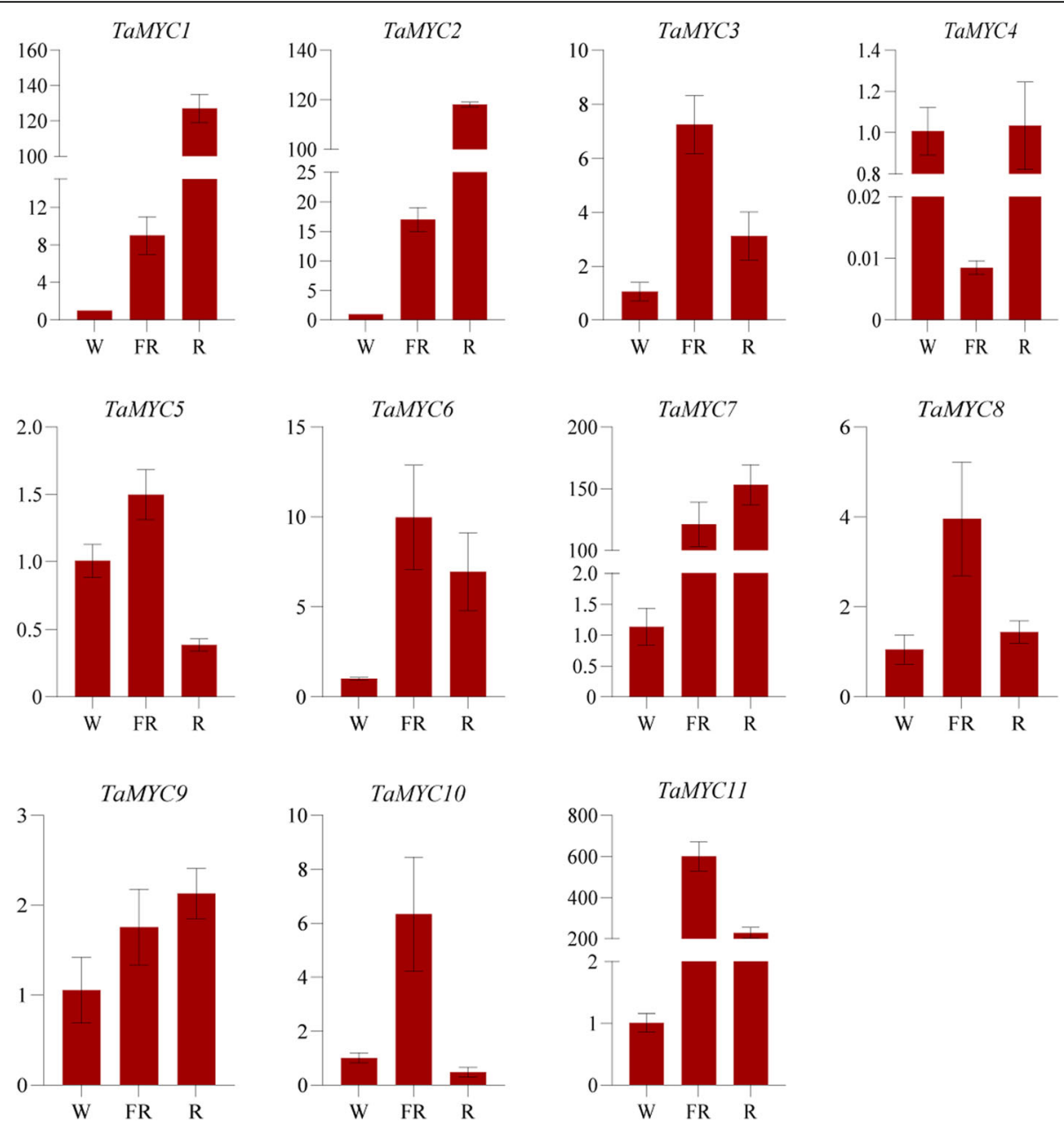

Fig. 7 Relative expression of TaMYC genes in anthers treated with different lights. The error bars indicate the standard deviation obtained from three replications. Wheat Actin gene is used as the inner reference

protein structures (Fig. 3), were up-regulated by treatments of ABA, GA, low temperatures, and drought (Fig. 8 and Fig.9). Similar expression profile trends also exited in TaMYC1 and TaMYC2 under treatment with low temperature and salinity treatments (Fig. 9).

Phytohormone crosstalk involves a very complex signaling regulative network, is universally available in plants, and plays a very important role in plant regulation of growth and development as the role of in JA signaling pathway [27]. TaMYC genes were found to contain different numbers of hormone-responsive elements, especially ABA (ABRE) and MeJA (CGTCA/ TGACG-motif) (Additional file 5: Table S2). ABA is a "stress hormone" that can regulate plant growth, stress tolerance, seed germination, and tissue/organ senescence [18]. In Arabidopsis, $M Y C 2$ functions in ABA signaling pathways [16]. In this study, TaMYC2, 4, 5, 6, and 8 were upregulated under ABA treatment (Fig. 8). It is known that MeJA may play a positive regulatory role in anther dehiscence and glume opening [2, 28]. As an important component of the JA signaling pathway, TaMYCs were also regulated by MeJA. For example, the promoter region of TaMYC3 was rich in CGTCA/ TGACG-motifs (numbers of motifs were 2, 1, and 3 for TaMYC3a-A, TaMYC $3 b-A$, and TaMYC3-B, respectively) (Fig. 4 and Additional file 5: Table S2), and they displayed very high expression levels after MeJA treatment (Fig. 6). Meanwhile, the transcriptional levels of the rest of TaMYC were significantly induced by different concentrations of MeJA (Fig. 6), although there were no CGTCA/TGACG-motifs in the promoter region of TaMYC4, 5 and 10. This indicated that the gene expression level under different treatments was not only dependent on the presence of relevant cis-acting regulatory elements, but also might be regulated by other physiological pathways in wheat.

A growing body of evidences indicate that the JA response is modulated by the ecological context of the plant, especially light, which is emerging as a critical regulator of JA signaling [29]. In Arabidopsis, MYC2 can 


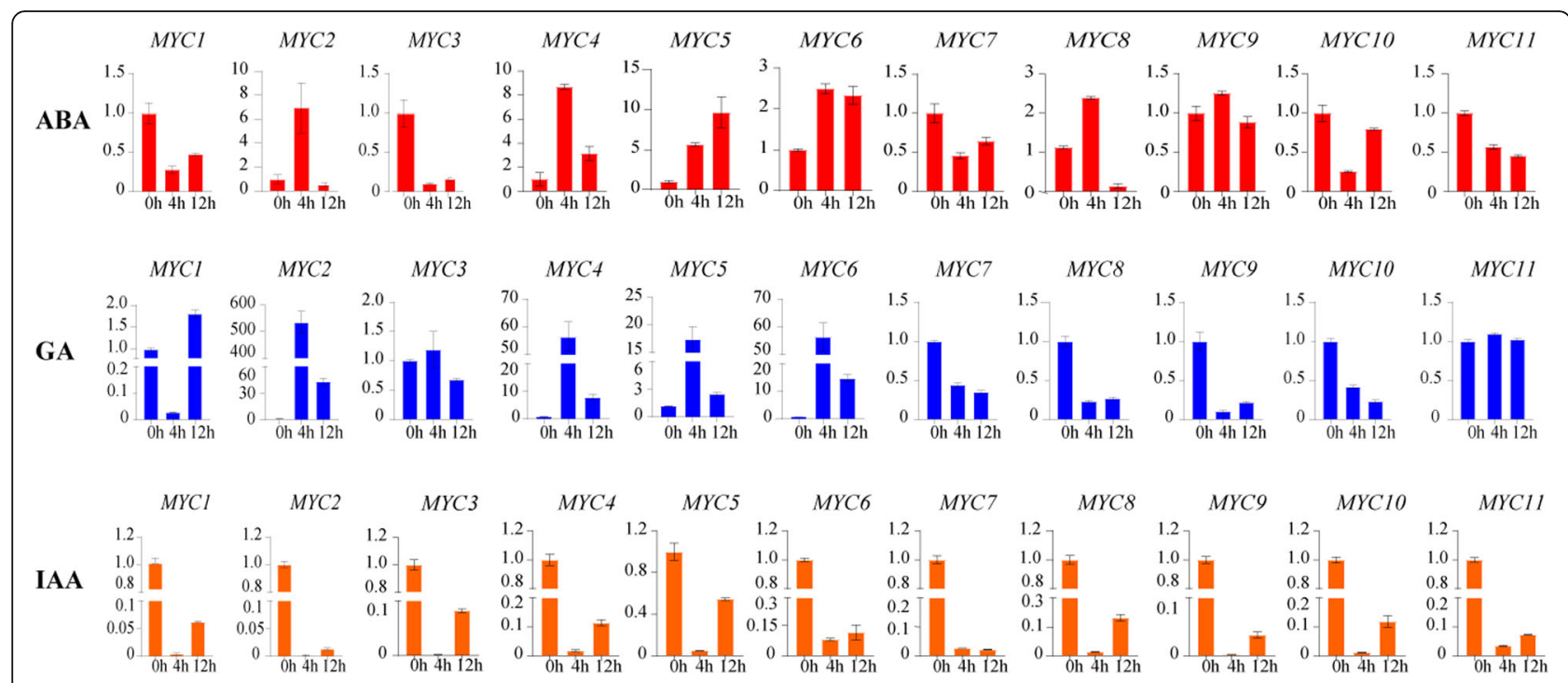

Fig. 8 Relative expression of TaMYC genes under phytohormone treatments. The error bars indicate the standard deviation obtained from three replications. Wheat actin gene is used as the inner reference

interact with SPA1 (suppressor of PHYA) redundantly in the dark and synergistically in the light to suppress photomorphogenesis by binding to the G-box (found in the promoter of SPA1) [21]. In this study, we also found that most of TaMYC genes were rich in G-box cis-acting regulatory elements. For example, TaMYC1, TaMYC5, TaMYC6, and TaMYC11 contained at least 4 G-box motifs, which are involved in light responsiveness (Fig. 4 and Additional file 5: Table S2). The state of conversion between Pfr and Pr is involved in detecting the quality of light by monitoring R:FR ratios [30]. Some evidences show that FR light-enriched environments could inactivate $\mathrm{PHYB}$ and promote the shade avoidance syndrome
[30]. To investigate the possible functions of TaMYCs in light signaling, we utilized the R: FR ratio-based $\mathrm{Pfr} / \mathrm{Pr}$ conversion experiment. The results showed that all TaMYCs were induced by $\mathrm{R}$ and $\mathrm{FR}$, such as the expression of TaMYC1, 2, 3, 6, 7, 8, 9 and 11, which were upregulated under both low and high R:FR ratios in the dark (Fig. 7). Here, the expression of TaMYC4 was downregulated under low R:FR ratios (far-red light-enriched environment), and expression of TaMYC5 was downregulated under high R:FR ratios (red light-enriched environment), indicating that these two genes might be regulated by other crosstalk pathways in wheat line BS366. In coil mutants, the expressions of JA biosynthetic gene (e.g.

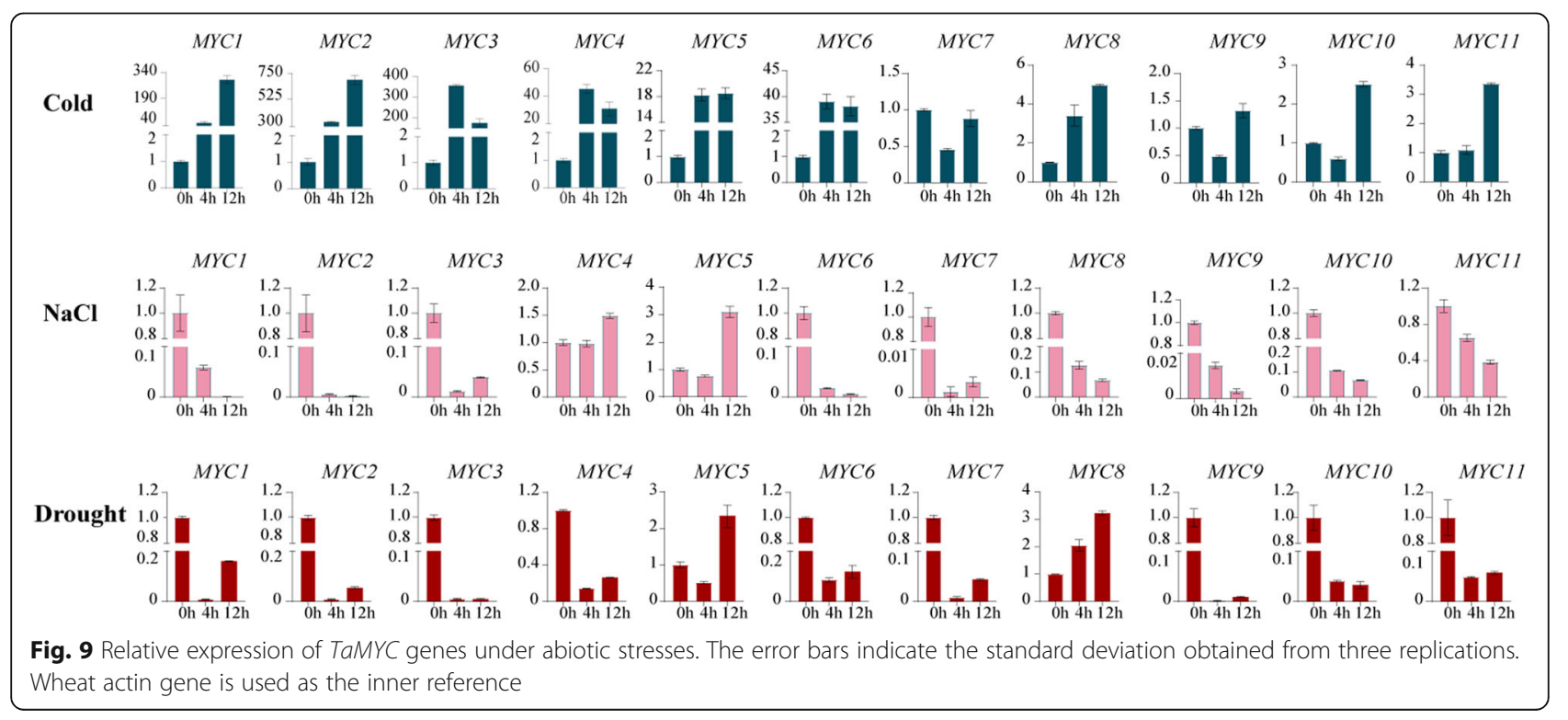




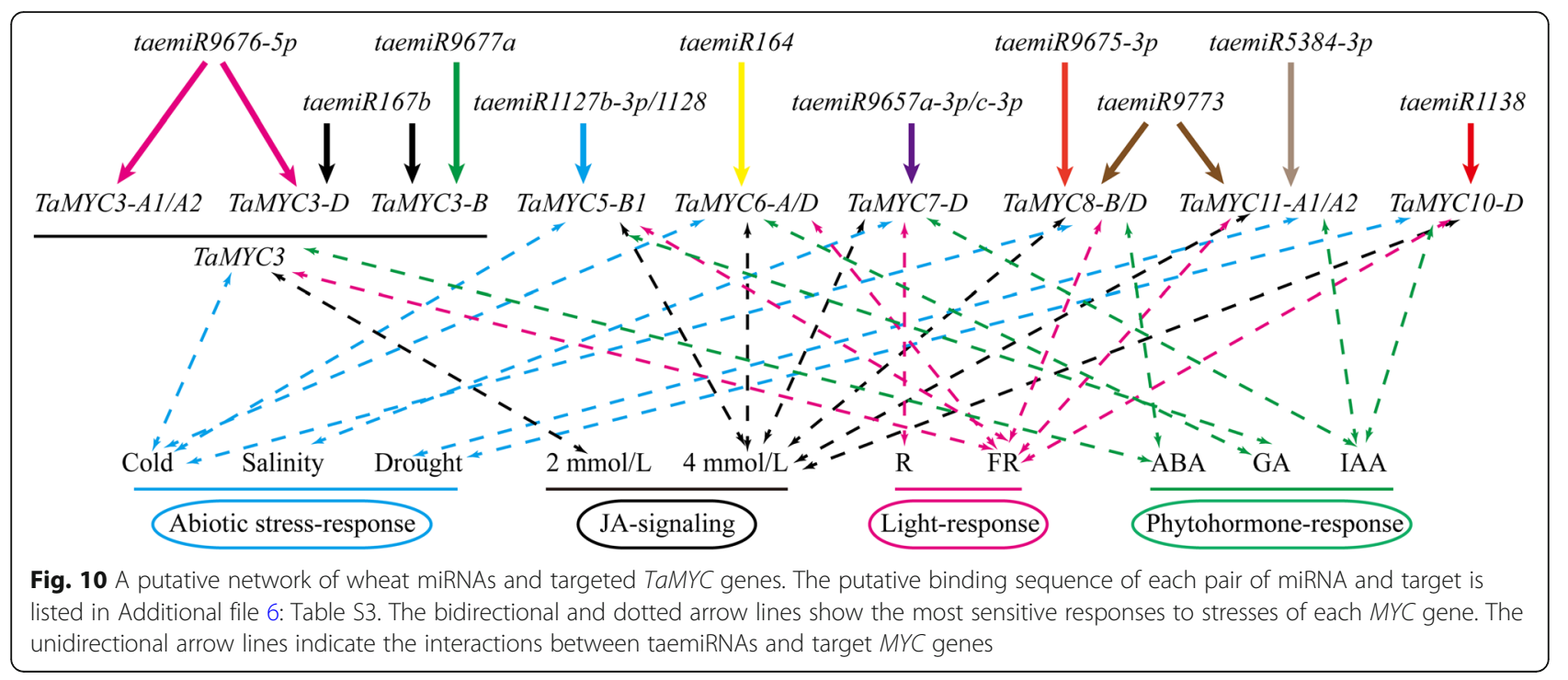

$A O C 1)$, signaling gene (JAZ1 and $M Y C 2)$, and wound response (VSP1) genes were attenuated, suggesting that FR light is a positive regulator of JA-responsive gene expression [13]. In this study, TaMYC genes showed different expression patterns under FR or R light-enriched environments, indicating they might differentially regulate different branches of the JA signaling pathway.

Many recent studies have indicated that miRNAs are involved in anther development and male sterility [19]. To further understand the roles of TaMYCs, the interaction relationship between TaMYCs and wheat miRNAs were predicted. Totally, seven TaMYCs were targeted by 12 taemiRNAs, including taemiR164 and taemiR167, which play important roles in plant growth and development (Fig. 10). In plants, miR164 targeted genes participate in various physiological and biochemical processes during plant development and in response to biotic/abiotic stress [31, 32]. For example, miR164 is involved in age-dependent cell death in Arabidopsis leaves by cleaving ORE (A NAM transcription factor) [33]. Plants with mutated miR164-CUC1 and miR164-CUC2 exhibit multiple phenotypes, such as leaf shape and polarity defects, extra petals, missing sepals, and reduced fertility [34, 35]. In addition, miR164 can also be regulated by light, and its expression was up-regulated under UV-B radiation treatment in maize [36]. In the present study, TaMYC6 responded to red/far-red light stimulation, hormone treatment, low temperatures stress, and drought stress, and might be targeted by miR164 (Fig. 10). In Arabidopsis, miR167 targeting Auxin Response Factor 6 (ARF6), and $A R F 8$, which regulates jasmonate biosynthesis by inhibiting downstream genes, regulates pollen development [37]. Wang et al. (2019) demonstrate that taemiR167, can induce male sterility and reduce the expression levels of AtARF6 and AtARF8 as well as reduce the content of JA and IAA in transgenic Arabidopsis [38]. One study shows that miR167 expression is upregulated in the light and downregulated in darkness, suggesting that miR167 may be regulated by light or the circadian clock [39]. Here, the results indicate that miR167 may interact with TaMYC3 (Fig. 10). With an experiment under MeJA treatment (Fig. 6), it was speculated that TaMYC3 might be a core factor in JA-light crosstalk, which needs to be verified by further experiments.

\section{Conclusions}

In this study, a comprehensive overview of the $M Y C$ gene family in wheat, including gene structures, phylogenetic relationships, and expression profiles, was provided. The roles of TaMYCs in response to abiotic stresses and light quality conversion were preliminary revealed. Moreover, our results provided insights into the crosstalk between MeJA signaling and light signaling in PTGMS wheat line BS366.

\section{Methods}

Plant materials, growth conditions, and sample collection Wheat PTGMS line BS366 (winter wheat) were planted in the experimental fields in Beijing (China, N 39 54', E $116^{\circ} 18^{\prime}$ ) and managed conventionally.

For tissue-specific expression analysis, wheat seedlings were grown until reaching the heading stage in experimental fields. Root, stem, leaf, petal, pistil, stamen, and glume of wheat were collected for tissue-specific expression analysis.

For abiotic stresses, two-week-old wheat seedlings were cultured in the thermostatic artificial climate chamber (CLC-BIV-M/CLC404-TV, MMM, Germany) $\left(20^{\circ} \mathrm{C}, 12 \mathrm{~h}\right.$ day $/ 12 \mathrm{~h}$ night cycle). For low temperatures stress, seedlings were placed in an incubator at $10{ }^{\circ} \mathrm{C}(12 \mathrm{~h}$ day $/ 12 \mathrm{~h}$ night cycle). For salinity and drought stresses, seedlings were treated with $200 \mathrm{mmol} / \mathrm{L} \mathrm{NaCl}$ solution and $20 \%$ 
PEG $6000(-0.5 \mathrm{MPa})$, respectively. For hormone treatments, seedlings were sprayed with $100 \mathrm{mmol} / \mathrm{L}$ ABA, 50 $\mathrm{mmol} / \mathrm{L} \mathrm{IAA}$, or $100 \mathrm{mmol} / \mathrm{L} \mathrm{GA}$, with water as the control. Leaf tissue was collected from seedlings after 0,4 and $12 \mathrm{~h}$ of stress treatment.

For effect of exogenous MeJA analysis, anthers of seedlings planted in experimental fields were treated with 0,2 and $4 \mathrm{mmol} / \mathrm{L} \mathrm{MeJA}$ solution at the heading stage. This experiment was carried out at the same time point within 5 days. Then collected at the anthers to investigate the effect of exogenous MeJA on the expression patterns of MYCs.

For effect of light quality conversation, four-leaf stage seedlings cultured in experimental fields were transferred into an artificial climate incubator with a 12-h photoperiod at a light intensity of $600 \mu \mathrm{mol} /\left(\mathrm{m}^{2 *} \mathrm{~s}\right)$ using fluorescent lamps. Night broken was performed at middle night $(\mathrm{ZT}=18 \mathrm{~h})$ using red $(\lambda=660 \mathrm{~nm}, 50 \mu \mathrm{mol} /$ $\left.\left(\mathrm{m}^{2 * \mathrm{~s}}\right)\right)$ and far-red light $\left(\lambda=731 \mathrm{~nm}, 40 \mu \mathrm{mol} /\left(\mathrm{m}^{2 *} \mathrm{~s}\right)\right)$ for ten days. Anthers were collected to determine the effect of conversion between red and far red lights on TaMYCs expression patterns. All samples were rapidly frozen in liquid nitrogen and stored at $-80^{\circ} \mathrm{C}$.

\section{Identification of the MYC gene family in wheat}

The genome sequence data and the annotation information of wheat (Chinese spring) were obtained from the Ensembl Plants database (http://plant.ensembl.org/index. html). MYC protein data of Arabidopsis thaliana (At) and Oryza sativa (Os) were downloaded from TIAR (http://www.Arabidopsis.org/) and TIGR (http://www. jcvi.org/).The hidden Markov models (HMM) of MYC protein (Pfam accessions: PF14215 and PF00010) were downloaded from the Pfam database (http://pfam.xfam. org/) [40], and were used as queries to search for potential MYC proteins in the wheat protein data sets by using HMMER3.0 [41] with an E-value cutoff of 1.0E-05. Subsequently, the nucleotide and genomic sequences of each TaMYC gene copy was confirmed based on the database accession number of protein. In order to confirm conserved domains of MYC, candidate protein sequences were subjected to online domain analysis program NCBI-CDD (https://www.ncbi.nlm.nih.gov/ cdd/), and SMART (http://smart.emblheidelberg.de/).

\section{Naming scheme of wheat MYC gene family}

$M Y C$ gene copies isolated in this study were defined as TaMYC. The different copies of same TaMYC gene in A, B, and D sub-genomes were clustered based on the phylogenetic results. The order of each TaMYC gene was decided based on their locations on A, B, and D wheat chromosomes and the clustering relationship on the cladogram.

\section{Chromosomal locations and synteny analysis}

Positional information of TaMYC genes were collected based on the genome annotation information. The synteny analysis of TaMYC genes was performed by using the whole genome synteny block data, which was available in the Plant Genome Duplication Database (http://chibba. agtec.uga.edu/duplication/) [42]. The visualization of chromosomal locations of TaMYC gene family was carried out by using Circos-0.69 (http://circos.ca/) [43].

\section{Physicochemical characteristics and cis-elements analysis}

Protein sequence length, theoretical isoelectric point and molecular weight of TaMYC proteins were predicted by using the ExPASy online tool (http://www.expasy.org/). Subcellular localization were predicted by using the WoLEPSORT online tool (http://www.genscript.com/ wolf-psort.html). To investigate putative cis-acting regulatory elements in the promoter regions of TaMYC genes, 1500 base pair genomic DNA sequences upstream of the initiation codon were retrieved and screened against the Plant CARE database (http://bioinformatics. psb.ugent.be/webtools/plantcare /html/) [44].

\section{Structural analysis of TaMYC genes and proteins}

The full-length coding sequences (CDS) and genomic DNA sequences of the TaMYC genes were collected from wheat reference genome database (IWGSC; https://www. wheatgenome.org/). The structure analysis of TaMYC genes were performed via GSDS2.0 (http://gsds.cbi.pku. edu.cn/) [45]. Conserved motifs of TaMYC proteins were analyzed using the online program MEME [46].

\section{Multiple sequence alignment and phylogenetic tree construction}

Multiple alignment of MYC protein sequences of wheat and other species (O. sativa and A. thaliana) was performed by using DNAMAN (ver. 6.0) with default parameters. An unrooted phylogenetic tree was constructed by using MEGA7.0 [47] with the neighbor-joining (NJ) method (1000 bootstrap trials, the Poisson model) based on the results of multiple alignment.

\section{Expression analysis of TaMYC genes}

Total RNA extraction, first-strand cDNA synthesis and Quantitative real-time PCR (qRT-PCR) was performed according to Bai et al. (2018) [2]. The data were analyzed using the $2^{-\Delta \Delta \mathrm{Ct}}$ method [48]. Each experiments were replicated three times. The primers used in this study were listed in Additional file 4: Table S1. Wheat actin gene (Genbank: AB181991) was used as the inner reference.

miRNA targeting prediction of the TaMYC gene family To investigate whether miRNA interacts with TaMYC genes, the known and studied miRNA sequences of 
wheat were collected from miRbase and subjected to online psRNATarget.

\section{Supplementary information}

Supplementary information accompanies this paper at https://doi.org/10. 1186/s12864-019-6373-y.

Additional file 1: Figure S1. Chromosomal localizations and syntenic relationships among TaMYC genes in Triticum aestivum, T. urartu and Ae Tauschii. Lines in grey indicate tandem duplication. Lines in blue, green and orange indicate segmental duplication

Additional file 2: Figure. S2. Consensus sequence and logos of motifs from wheat MYC proteins

Additional file 3: File S1. Promoter sequences of MYC gene copies

Additional file 4: Table S1. The primers used in this study

Additional file 5: Table S2. Numbers of cis-regulatory elements in the upstream promoter regions of TaMYC genes

Additional file 6: Table S3. miRNA targeting prediction of TaMYC gene family

Additional file 7: Table S4. Segmental duplication of TaMYC genes

\section{Abbreviations}

ABA: Abscisic acid; GA: Gibberellin; IAA: Indole-3-acetic acid; MeJA: Methyl jasmonate; PTGMS: Photoperiod-Temperature sensitive Genic Male Sterile

\section{Acknowledgements}

We are grateful to the reviewers for their helpful suggestions. We thank editors very much for their efficient works.

\section{Authors' contributions}

$J B, Y W, L Z$ and $C Z$ designed the study. YW, LG and JB performed the experiments, analyzed the data, and drafted the manuscript. $Z L, H G, X G$ and WD assisted with bioinformatics analysis and aided in writing the manuscript. SY, HG, XG and FZ aided in performing the experiments.

\section{Funding}

This study was supported by the National Natural Science Foundation of China (31872881); Outstanding Scientist Cultivation Program of BAAFS (JKZX201907); Special Project of Science and Technology Innovation Ability Construction of BAAFS (KJCX20180403); National Science Foundation for Young Scientists of China (31901490); Natural Science Foundation of Beijing (6182014).

\section{Availability of data and materials}

The data sets supporting the results of this article are included within the article and its additional files.

\section{Ethics approval and consent to participate}

PTGMS wheat line BS366 seeds were provided by Beijing Engineering and Technique Research Center for Hybrid Wheat.

\section{Consent for publication}

Not applicable.

\section{Competing interests}

The authors declare that they have no competing interests.

\section{Author details}

'Beijing Engineering Research Center for Hybrid Wheat, Beijing Academy of Agriculture and Forestry Sciences, Beijing 100097, China. ${ }^{2}$ The Municipal Key Laboratory of Molecular Genetic of Hybrid Wheat, Beijing 10097, China. ${ }^{3}$ Division of Biological Science, Graduate School of Science and Technology, Nara Institute of Science and Technology, Nara 630-0192, Japan. ${ }^{4}$ School of Landscape Architecture, Beijing Forestry University, Beijing 100083, People's Republic of China.
Received: 5 October 2019 Accepted: 5 December 2019

Published online: 30 December 2019

\section{References}

1. Schweizer F, Fernãn-CP, Zander M, Diez-Diaz M, Fonseca S, Glauser G, Lewsey MG, Ecker JR, Solano R, Reymond P. Arabidopsis basic helix-loophelix transcription factors MYC2, MYC3, and MYC4 regulate glucosinolate biosynthesis, insect performance, and feeding behavior. Plant Cell. 2013; 25(8):3117-32

2. Bai JF, Wang YK, Wang P, Yuan SH, Gao JG, Duan WJ, Wang N, Zhang FT, Zhang WJ, Qin MY, et al. Genome-wide identification and analysis of the COl gene family in wheat (Triticum aestivum L.). BMC genomics. 2018;19(1):754.

3. Chini A, Boter M, Solano R. Plant oxylipins: COI1/JAZs/MYC2 as the core jasmonic acid-signalling module. FEBS J. 2009;276(17):4682-92.

4. Pauwels L, Barbero GF, Geerinck J, Tilleman S, Grunewald W, Perez AC Chico JM, Bossche RV, Sewell J, Gil E, et al. NINJA connects the co-repressor TOPLESS to jasmonate signalling. Nature. 2010;464(7289):788-91.

5. Cheng Z, Sun L, Qi T, Zhang B, Peng W, Liu Y, Xie D. The bHLH transcription factor MYC3 interacts with the jasmonate ZIM-domain proteins to mediate jasmonate response in Arabidopsis. Mol Plant. 2011;4(2):279-88.

6. Fernandez-Calvo P, Chini A, Fernandez-Barbero G, Chico J-M, GimenezIbanez S, Geerinck J, Eeckhout D, Schweizer F, Godoy M, Franco-Zorrilla JM. The Arabidopsis bHLH transcription factors MYC3 and MYC4 are targets of JAZ repressors and act additively with MYC2 in the activation of jasmonate responses. Plant Cell. 2011;23(2):701

7. Lorenzo O, Chico JM, Sanchez-Serrano JJ, Solano R. JASMONATEINSENSITIVE1 encodes a MYC transcription factor essential to discriminate between different jasmonate-regulated defense responses in Arabidopsis. Plant Cell. 2004;16(7):1938-50.

8. Dombrecht B, Xue GP, Sprague SJ, Kirkegaard JA, Ross JJ, Reid JB, Fitt GP, Sewelam N, Schenk PM, Manners JM, et al. MYC2 differentially modulates diverse jasmonate-dependent functions in Arabidopsis. Plant Cell. 2007;19(7):2225-45.

9. Chico J, Fernándezbarbero G, Chini A, Fernándezcalvo P, Díezdíaz M, Solano R. Repression of jasmonate-dependent defenses by shade involves differential regulation of protein stability of MYC transcription factors and their JAZ repressors in Arabidopsis. Plant Cell. 2014;26(5):1967-80.

10. Folta KM, Maruhnich SA. Green light: a signal to slow down or stop. J Exp Bot. 2007;58(12):3099.

11. Martínez-García JF, Galstyan A, Salla-Martret M, Cifuentes-Esquivel N, Gallemí M, Bou-Torrent J. Regulatory components of shade avoidance syndrome. Adv Bot Res. 2010;53:65-116.

12. Kazan $\mathrm{K}$, Manners JM. The interplay between light and jasmonate signalling during defence and development. J Exp Bot. 2011;62(12):4087-100.

13. Robson F, Okamoto H, Patrick E, Harris SR, Wasternack C, Brearley C, Turner JG. Jasmonate and phytochrome a signaling in Arabidopsis wound and shade responses are integrated through JAZ1 stability. Plant Cell. 2010;22(4): 1143-60.

14. Yadav V, Mallappa C, Gangappa SN, Bhatia S, Chattopadhyay S. A basic helix-loop-helix transcription factor in Arabidopsis, MYC2, acts as a repressor of blue light-mediated photomorphogenic growth. Plant Cell. 2005;17(7): 1953-66.

15. Gangappa SN, Chattopadhyay S. MYC2, a bHLH transcription factor, modulates the adult phenotype of SPA1. Plant Signal Behav. 2010;5(12):1650-2.

16. Abe H, Urao T, Ito T, Seki M, Shinozaki K, Yamaguchi-Shinozaki K. Arabidopsis AtMYC2 (bHLH) and AtMYB2 (MYB) function as transcriptional activators in abscisic acid signaling. Plant Cell. 2003;15(1):63-78.

17. Song S, Huang H, Gao H, Wang J, Wu D, Liu X, Yang S, Zhai Q, Li C, Qi T, et al. Interaction between MYC2 and ETHYLENE INSENSITIVE3 modulates antagonism between jasmonate and ethylene signaling in Arabidopsis. Plant Cell. 2014;26(1):263-79.

18. Wang Y, Qiao L, Bai J, Wang P, Duan W, Yuan S, Yuan G, Zhang F, Zhang L, Zhao C. Genome-wide characterization of JASMONATE-ZIM DOMAIN transcription repressors in wheat (Triticum aestivum L.).BMC Genomics. 2017;18(1):152.

19. Bai JF, Wang YK, Wang P, Duan WJ, Yuan SH, Sun H, Yuan GL, Ma JX, Wang $\mathrm{N}$, Zhang $\mathrm{FT}$, et al. Uncovering male fertility transition responsive miRNA in a wheat photo-thermosensitive genic male sterile line by deep sequencing and degradome analysis. Front Plant Sci. 2017;8:1370

20. Wu Y, Zhu Z, Ma L, Chen M. The preferential retention of starch synthesis genes reveals the impact of whole-genome duplication on grass evolution. Mol Biol Evol. 2008;25(6):1003-6. 
21. Gangappa SN, Prasad VB, Chattopadhyay S. Functional interconnection of MYC2 and SPA1 in the photomorphogenic seedling development of Arabidopsis. Plant Physiol. 2010;154(3):1210-9.

22. Turner JG, Ellis C, Devoto A. The jasmonate signal pathway. Plant Cell. 2002; 14(14 Suppl):S153.

23. Shan X, Zhang Y, Peng W, Wang Z, Xie D. Molecular mechanism for jasmonate-induction of anthocyanin accumulation in Arabidopsis. J Exp Bot. 2009;60(13):3849-60.

24. Reinbothe C, Springer A, Samol I, Reinbothe S. Plant oxylipins: role of jasmonic acid during programmed cell death, defense and leaf senescence. FEBS J Febs Journal. 2010;276(17):4666-81.

25. Wittkopp PJ, Kalay G. Cis-regulatory elements: molecular mechanisms and evolutionary processes underlying divergence. Nat Rev Genet. 2012;13(1): 59-69.

26. Nakashima K, Ito Y, Yamaguchi-Shinozaki K. Transcriptional regulatory networks in response to abiotic stresses in Arabidopsis and grasses. Plant Physiol. 2009;149(1):88-95.

27. Kazan K, Manners JM. JAZ repressors and the orchestration of phytohormone crosstalk. Trends Plant Sci. 2012;17(1):22-31.

28. Liu SY, Qin ZL, Zhang FT, Zhao CP, Qing MA. Hormonal regulation of glume opening and closure of photo-thermo-sensitive male sterility wheat by methyl jasmonate and salicylic acid. J Triticeae Crops. 2007;27(4):593-596.

29. Radhika V, Kost C, Mithofer A, Boland W. Regulation of extrafloral nectar secretion by jasmonates in lima bean is light dependent. Proc Natl Acad Sci U S A. 2010;107(40):17228-33.

30. Martínez-García, J F, Galstyan A, Salla-Martret M, Cifuentes-Esquivel N, Gallemí M, Bou-Torrent J. Regulatory components of shade avoidance syndrome. Adv Bot Res. 2010;53:66-116.

31. Zheng L, Zhang $X$, Zhang H, Gu Y, Huang X, Huang H, Liu H, Zhang J, Hu Y, Li Y, Yu G, Liu Y, Lawson SS, Huang Y. The miR164-dependent regulatory pathway in developing maize seed. Molecular genetics and genomics : MGG. 2019;294(2):501-17.

32. Fang $Y$, Xie K, Xiong L. Conserved miR164-targeted NAC genes negatively regulate drought resistance in rice. J Exp Bot. 2014;65(8):2119-35.

33. Kim JH, Woo HR, Kim J, Lim PO, Lee IC, Choi SH, Hwang D, Nam HG. Trifurcate feed-forward regulation of age-dependent cell death involving miR164 in Arabidopsis. Science. 2009;323(5917):1053-7.

34. Laufs P. MicroRNA regulation of the CUC genes is required for boundary size control in Arabidopsis meristems. Development. 2004;19(1):4311-22.

35. Mallory AC, Dugas DV, Bartel DP, Bartel B. MicroRNA regulation of NACdomain targets is required for proper formation and separation of adjacent embryonic, vegetative, and floral organs. Curr Biol. 2004;14(12):1035-46.

36. Casati $P$. Analysis of UV-B regulated miRNAs and their targets in maize leaves. Plant Signal Behav. 2013;8(10):-e26758.

37. Tabata R, Ikezaki M, Fujibe T, Aida M, Tian CE, Ueno Y, Yamamoto KT, Machida $Y$, Nakamura $K$, Ishiguro S. Arabidopsis auxin response factor6 and 8 regulate jasmonic acid biosynthesis and floral organ development via repression of class 1 KNOX genes. Plant \& cell physiology. 2010;51(1):164-75.

38. Wang Y, Duan W, Bai J, Wang P, Yuan S, Zhao C, Zhang L. Constitutive expression of a wheat microRNA, TaemiR167a, confers male sterility in transgenic Arabidopsis. Plant Growth Regul. 2019;88(3):227-39.

39. Sire C, Moreno AB, Garcia-Chapa M, Lopez-Moya JJ, San Segundo B. Diurnal oscillation in the accumulation of Arabidopsis microRNAs, miR167, miR168, miR171 and miR398. FEBS Lett. 2009;583(6):1039-44.

40. Finn RD, Coggill P, Eberhardt RY, Eddy SR. The Pfam protein families database: towards a more sustainable future. Nucleic Acids Res. 2016; 44(D1):D279-85.

41. Eddy SR. A probabilistic model of local sequence alignment that simplifies statistical significance estimation. PLoS Comput Biol. 2008;4(5):e1000069.

42. Lee $\mathrm{TH}$, Tang H, Wang $X$, Paterson AH. PGDD: a database of gene and genome duplication in plants. Nucleic Acids Res. 2013;41(Database issue): D1152-8.

43. Krzywinski M, Schein J, Birol I, Connors J, Gascoyne R, Horsman D, Jones SJ, Marra MA. Circos: an information aesthetic for comparative genomics. Genome Res. 2009;19(9):1639-45.

44. Lescot M, Dehais P, Thijs G, Marchal K, Moreau Y, Van de Peer Y, Rouze P, Rombauts S. PlantCARE, a database of plant cis-acting regulatory elements and a portal to tools for in silico analysis of promoter sequences. Nucleic Acids Res. 2002;30(1):325-7.

45. Hu B, Jin J, Guo AY, Zhang H, Luo J, Gao G. GSDS 2.0: an upgraded gene feature visualization server. Bioinformatics. 2015;31(8):1296-7.
46. Bailey TL, Boden M, Buske FA, Frith M, Grant CE, Clementi L, Ren J, Li WW, Noble WS. MEME SUITE: tools for motif discovery and searching. Nucleic acids research. 2009;37(Web Server issue):W202-8.

47. Kumar S, Stecher G, Tamura K. MEGA7: molecular evolutionary genetics analysis version 7.0 for bigger datasets. Mol Biol Evol. 2016;33(7):1870-4.

48. Livak KJ, Schmittgen TD. Analysis of relative gene expression data using real-time quantitative PCR and the 2(-Delta Delta C(T)) Method. 2001; 25(Methods, 4):402-8.

\section{Publisher's Note}

Springer Nature remains neutral with regard to jurisdictional claims in published maps and institutional affiliations.
Ready to submit your research? Choose BMC and benefit from:

- fast, convenient online submission

- thorough peer review by experienced researchers in your field

- rapid publication on acceptance

- support for research data, including large and complex data types

- gold Open Access which fosters wider collaboration and increased citations

- maximum visibility for your research: over $100 \mathrm{M}$ website views per year

At BMC, research is always in progress.

Learn more biomedcentral.com/submissions 